\title{
Multimodal management of malignant pleural mesothelioma: where are we today?
}

\author{
Paul E. Van Schil', Isabelle Opitz², Walter Weder ${ }^{2}$, Christophe De Laet ${ }^{1}$, \\ Andreas Domen ${ }^{1}$, Patrick Lauwers ${ }^{1}$, Jeroen M. Hendriks ${ }^{1}$, and \\ Jan P. Van Meerbeeck ${ }^{3}$
}

\author{
Number 4 in the series "Challenges and Controversies in Thoracic Oncology" \\ Edited by J-P. Sculier, B. Besse and P. Van Schil
}

Affiliations: ${ }^{1}$ Dept of Thoracic and Vascular Surgery, Antwerp University Hospital, Antwerp, Belgium. ${ }^{2}$ Division of Thoracic Surgery, University Hospital, Zurich, Switzerland. ${ }^{3}$ Multidisciplinary Oncological Centre Antwerp, Division of Thoracic Oncology, Antwerp University Hospital, Antwerp, Belgium.

Correspondence: Paul E. Van Schil, Dept of Thoracic and Vascular Surgery, Antwerp University Hospital, Wilrijkstraat 10, B-2650 Edegem (Antwerp), Belgium. E-mail: paul.van.schilauza.be

ABSTRACT The prognosis of patients with malignant pleural mesothelioma remains poor and although it is clear that multimodal therapy is necessary to improve long-term results, precise treatment schemes have not yet been unequivocally established. Single-modality therapy does not have a major impact on long-term survival and combined-modality therapies are being further evaluated. However, the relative contributions of chemotherapy, radiotherapy and surgery have not been clearly determined at the present time. Moreover, the extent of resection and precise surgical procedure remain a highly debated topic. To better compare and combine results from different institutions and trials, uniform definitions of surgical procedures including extrapleural pneumonectomy and different forms of pleurectomy have recently been introduced. Due to the relatively higher morbidity and mortality of extrapleural pneumonectomy, there is currently a shift towards pleurectomy/decortication when a macroscopic complete resection of all tumour can be obtained by this procedure. In most recent trials, induction chemotherapy was administered to improve surgical resection rates but pathological complete responses are infrequently observed. The role of post-operative radiotherapy has to be further elucidated.

Further treatment options that are currently explored include hyperthermic intrapleural chemotherapy, immunotherapy, gene therapy and photodynamic therapy. However, no randomised comparisons are available yet.

@ERSpublications

Treatment of malignant pleural mesothelioma remains controversial http://ow.ly/sNCY1

\footnotetext{
Previous articles in this series: No. 1: Powell HA, Baldwin DR. Multidisciplinary team management in thoracic oncology: more than just a concept? Eur Respir J 2014; 43: 1776-1786; No. 2: Shlomi D, Ben-Avi R, Balmor GR, et al. Screening for lung cancer: time for large-scale screening by chest computed tomography. Eur Respir J 2014; 44: 217-238; No. 3: De Ruysscher D, Nakagawa K, Asamura H. Surgical and nonsurgical approaches to small-size nononsmall cell lung cancer. Eur Respir J 2014; 44: 483-494.
}

Received: Nov 272013 | Accepted after revision: Dec 272013 | First published online: Feb 132014

Conflict of interest: None declared.

Copyright @ERS 2014 


\section{Introduction}

Malignant pleural mesothelioma (MPM) is an uncommon tumour arising from the parietal pleura and is characterised by a peculiar growth pattern extending along the pleural surface from the costodiaphragmatic sinus, where frequently a large amount of tumour is found, to the apical region of the chest. Subsequently, it expands into the lung parenchyma, chest wall, mediastinum and abdominal cavity. A causative link with asbestos exposure has been repeatedly documented and asbestos is considered a carcinogen [1]. The incidence of mesothelioma is expected to increase until the 2020s due to continuing use of asbestos and the long time interval between exposure and onset of the disease [2, 3]. As MPM is a non-spherical tumour, precise determination of tumour size and volume are difficult, limiting the validity of the current staging systems, of which the TNM (tumour, node, metastasis) classification published in 1995 by the International Mesothelioma Interest Group (IMIG) is the prevailing one [4, 5]. In the TNM system, only anatomical features are taken into consideration and, as precise anatomical landmarks are surgically driven, this system has several limitations because most patients with mesothelioma will not undergo extensive surgical therapy. However, precise clinical staging is important to determine subsequent prognosis and allocate tailored treatment to each individual patient. In contrast to lung cancer, clinical staging in mesothelioma is substantially more different from the final pathological stage, making the decision process problematic.

Accurate restaging is also difficult and tumour response can best be evaluated by comparing perpendicular diameters as proposed in the modified Response Evaluation Criteria in Solid Tumours [6]. These were recently optimised with only one cut-off value yielding two response categories, although further prospective validation is necessary [7]. Newer software allowing more precise tumour volume measurements will probably increase the staging accuracy in the near future [8,9]. Lung volume measurements can be considered a surrogate marker for patient response, as it has recently been demonstrated that increasing disease volume and decreasing lung volume are associated with a poor prognosis [10].

Even in early-stage disease, prognosis remains grim with an overall median survival time of 9-12 months [11]. For this reason, newer treatment modalities are currently being explored, mostly consisting of multimodality therapy comprising chemotherapy, radiotherapy and surgery $[12,13]$. The precise role and type of surgery remain highly controversial and no precise guidelines are currently available due to the lack of large randomised controlled trials [14]. This article describes the different types of multimodal therapy with an emphasis on the contribution of surgery to combined-modality therapy.

\section{Prognostic factors}

In the 1995 TNM classification, nodal staging was adopted from non-small cell lung cancer, although lymph node spread of MPM is quite different as the tumour originates from the parietal pleura, and intercostal, pericardial and mediastinal nodal involvement may occur rather early in the disease process, reflecting a local (N1) rather than a regional (N2-N3) lymphatic spread.

Several prognostic indices were developed taking different clinical features and laboratory values into account. Most widely used are the European Organisation for Research and Treatment of Cancer (EORTC) and Cancer and Leukemia Group B (CALGB) prognostic indices [2, 15, 16]. CALGB evaluated prognostic factors in 337 patients treated with chemotherapy for advanced-stage mesothelioma [16]. Poor prognostic factors in relation to survival were age $>75$ years, poor performance status, chest pain, nonepithelial histology, platelet count $\geqslant 4 \times 10^{14}$ per litre and lactate dehydrogenase concentration $\geqslant 500 \mathrm{IU} \cdot \mathrm{L}^{-1}$. The EORTC index is based on survival data of 204 patients who were entered in consecutive phase II trials [15]. Poor performance status, male sex, sarcomatoid subtype, white blood cell count $\geqslant 8.3 \times 10^{9}$ cells per litre and no definite diagnosis were identified as poor prognostic factors. Both indices were validated in subsequent trials. A prognostic nomogram allows estimation of overall and progression-free survival [17]. In a recent study of 88 patients with epitheloid mesothelioma who underwent extrapleural pneumonectomy, tumour volume, haemoglobin concentration and administration of adjuvant chemotherapy or radiotherapy were found to be independent factors associated with overall survival [18].

Recently, a risk score was developed to determine which patients would benefit from surgical therapy after induction chemotherapy [19]. In this so-called Zurich score, four variables are considered: initial tumour volume $>500 \mathrm{~mL}$, nonepithelial tumour type, initial C-reactive protein concentration $>30 \mathrm{mg} \cdot \mathrm{L}^{-1}$ and progressive disease after induction chemotherapy. Patients with three or four risk factors have a very poor prognosis and do not benefit from extensive surgical therapy. This score is currently validated in an independent second cohort.

To obtain more meaningful survival data on this rare tumour, the International Association for the Study of Lung Cancer (IASLC) retrospectively collected 3101 cases from 15 centres on four continents [13]. Currently, this is the largest international database of mesothelioma patients, which has recently been 
analysed [13]. Median age was 63 years and the epithelial subtype was present in $62.3 \%$ of patients. The socalled best TNM stages incorporating clinical and pathological staging information were stage I (11\% of patients in the database), II (21\%), III (48\%) and IV (20\%). In 1494 (64.5\%) patients, surgery with curative intent was undertaken. Median survival times according to stage were 21, 19, 16 and 12 months for stages I, II, III and IV, respectively with no difference between clinical and pathological stage. Regarding histology, median survival time for epithelial tumours was 19 months, for biphasic histology 13 months and for sarcomatoid tumours 8 months. In multivariable analysis, significant differences were found between T4 and T3 disease, and between T3 and T2, but not between T2 and T1. The difference between N0 and N1+N2 was also significant but not that between $\mathrm{N} 1$ and N2 disease. There was also a significant difference between stages III+IV compared with stage I, but not between stages I and II. Also significant were: epithelial type versus other tumour types; sex, with female patients having best prognosis; and finally, resection with curative intent versus palliative surgery. Patients with stage I disease treated by extrapleural pneumonectomy had a better prognosis compared with those undergoing pleurectomy/decortication. These are all very meaningful data but they should be interpreted with caution as they were retrospectively collected in surgically treated patients and subject to selection bias.

Subsequently, a prospective database has been launched by IASLC that is currently collecting data from all over the world in preparation for a new TNM classification [20]. In this way, more robust data will be obtained also including nonsurgical patients. Other prognostic factors will be taken into account (e.g. histological subtype) [21].

\section{Surgical treatment of mesothelioma}

The role of surgery for mesothelioma remains a highly controversial and intensely debated topic at major conferences and in the present-day literature. A distinction should be made between palliative surgical treatment and surgery with curative intent, so-called maximal debulking surgery.

\section{Palliative surgical treatment}

The thoracic surgeon plays an important role in the diagnosis, staging and palliative therapy of mesothelioma patients. To obtain large biopsy samples allowing detailed pathological and immunohistochemical analysis, video-assisted thoracic surgery (VATS) or thoracoscopy with double-lumen intubation and one-lung ventilation is an excellent procedure providing a high diagnostic yield, as extensive biopsies of parietal and visceral pleura can be taken. At the same time, a persistent pleural fluid effusion may be drained and dyspnoea relieved by performing a pleurodesis procedure, most commonly with talc spray, which is highly efficient on the condition that sufficient lung expansion is obtained. In addition, partial pleurectomy can be helpful in obtaining pleurodesis, as the slightly bleeding surface induces pleural adhesions [22]. One observational study on VATS pleurectomy suggested that this might be associated with improved survival [23].

In the recently analysed mesoVATS trial, 196 patients were randomised to receive either partial pleurectomy by VATS and talcage by chest tube or thoracoscopic poudrage [24]. Although overall survival was not improved in the VATS group, quality of life and control of pleural effusion were found to be significantly better in the VATS group after 6 and 12 months.

In patients with a trapped lung due to extensive visceral pleural and lung parenchymal involvement, continuous evacuation of pleural fluid may be achieved by an indwelling pleural catheter or a pleuroperitoneal shunt, the latter requiring active participation of the patient by pumping fluid from the pleural to the peritoneal cavity due to the pressure difference between both cavities [25, 26]. A pleuroperitoneal shunt entails a theoretical risk of tumour spread to the peritoneal cavity. However, this shunt is only inserted in patients who have a poor prognosis anyway; so, in daily clinical practice, this does not constitute a common problem. In a series of 160 patients, malignant seeding along the chest wall occurred in only one patient at the site of shunt insertion [25].

\section{Surgery with curative intent}

To remove as much tumour as possible, different surgical procedures exist in order to obtain a macroscopic complete resection or maximal debulking with the intent to improve local control and prolong disease-free survival. However, as the tumour originates on the parietal pleura; from a surgical point of view, it is not possible to obtain broad margins around it, especially on the outer margin close to the chest wall and costodiaphragmatic sinus. Over recent years, confusion has risen over the precise definitions of surgical procedures described in several series, especially the so-called "radical" pleurectomy and decortication. This makes comparison of results and outcomes between different centres quite tedious. For this reason, the IASLC created a working group to recommend uniform definitions for surgical procedures dealing with mesothelioma [27]. The confusing term "radical pleurectomy" is not used anymore as it implies that all tumour is removed and that no microscopic deposits remain, which is nearly impossible to obtain, except 

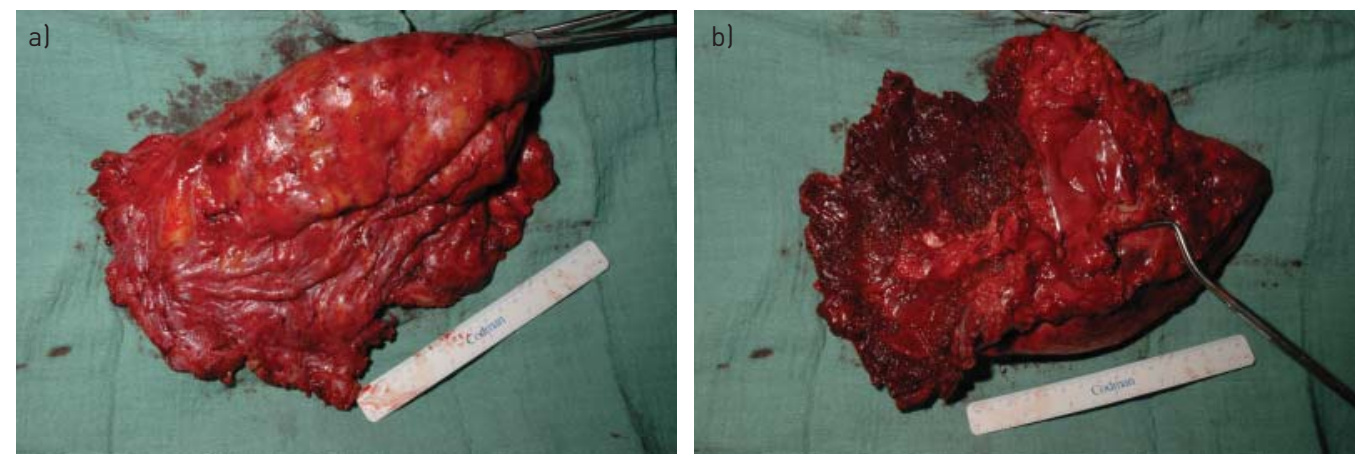

FIGURE 1 Extrapleural pneumonectomy comprising removal of parietal pleura, ipsilateral lung with visceral pleura, diaphragm and pericardium. a) Lateral view; b) hilar view.

for in very early stages. Currently, a clear distinction is made between extrapleural pneumonectomy (EPP) and pleurectomy/decortication (P/D) with different subcategories. EPP implies complete en bloc removal of the involved parietal and visceral pleura, the whole ipsilateral lung and, if necessary, the pericardium and diaphragm, which are reconstructed with a soft tissue patch (fig. 1).

Extended P/D comprises the same procedure but leaving the lung in situ. The pericardium and diaphragm are resected and reconstructed, if necessary. P/D refers to removal of all gross tumour without diaphragmatic or pericardial resection, whereas partial pleurectomy entails partial removal of parietal and/ or visceral pleura leaving gross tumour behind. It is clear that the latter procedure is performed for diagnostic or palliative reasons. When these definitions will be applied universally, better comparison between centres, specific trials involving surgery and international databases will become feasible.

\section{Combined-modality therapy}

Even 30 years ago, it was clear that MPM is difficult to treat and that, for most patients, there is no prospect of cure [28]. Single-modality therapy, be it surgery, radiotherapy, chemotherapy or immunotherapy, will not result in a substantial improvement in disease-free or overall survival. In the previous century, therapeutic nihilism prevailed for a long time. This changed when two randomised trials demonstrated that folate antagonists in combination with cisplatin significantly improved survival in unresectable patients, although the absolute survival benefit was limited to a few months only [29-31].

In the meantime, more experience was gained with extensive cytoreductive surgery for mesothelioma, especially EPP. In high-volume centres, mortality could be substantially reduced but morbidity remained relatively high, indicating that these procedures should be performed in centres with a large experience in thoracic surgery [32].

In a compiled series from three large institutions, 663 patients undergoing EPP or P/D between 1990 and 2006 were analysed [33]. Operative mortality was 7\% for EPP and 4\% for P/D. Significant factors related to survival were stage, epithelial cell type, type of resection, multimodality therapy and sex.

To improve local control, bimodal therapy administering high-dose radiotherapy after extensive surgical resection was introduced. This is more easily performed after EPP than P/D as there is an empty cavity without remaining lung parenchyma. In a phase II trial of 57 patients undergoing EPP or P/D, a postoperative dose of $54 \mathrm{~Gy}$ was administered [34]. Local recurrence was $13 \%$ with a median survival time of 17 months, which was lower than historical series. However, the precise dose and timing of radiotherapy remained unclear. Whether low-dose radiotherapy has to be delivered at incisional sites, including thoracoports, remains controversial, as conflicting results have been reported in recent literature [35-39]. A pooled analysis found no significant reduction in the frequency of procedure tract metastases [40].

Is trimodal therapy a valid option for patients with mesothelioma that is technically resectable? Different combined-modality regimens are under investigation. Similar to locally advanced lung cancer, induction chemotherapy was considered to increase the complete resection rate of early-stage mesothelioma.

In a Swiss multicentre trial, three cycles of cisplatin and gemcitabine were given as induction therapy followed by EPP and post-operative radiotherapy to incompletely resected areas [41]. In total, 61 patients with clinical T2N2M0 mesothelioma or less were included and 45 patients underwent EPP with or without post-operative radiotherapy. Macroscopic complete resection was obtained in 37 (61\%) patients and 36 had post-operative radiotherapy. 90 -day mortality was only $3.2 \%$ but one or more complications occurred in $62 \%$. The incidence of empyema (16\%) and bronchopleural fistula $(9.5 \%)$ were higher than that observed 
after EPP without induction therapy. For the patients undergoing EPP, an encouraging median survival time of 23 months was obtained.

Following the significant results of chemotherapy trials with anti-folate drugs, induction therapy comprising pemetrexed was evaluated to improve results with the hope of substantially increasing overall survival. In a retrospective study of trimodal therapy, 60 patients underwent induction chemotherapy (cisplatin/anti-folate in 30 patients), followed by EPP and post-operative radiotherapy to $\geqslant 50$ Gy [42]. The full protocol could be applied in 50\% of patients. Pathological nodal staging was a significant predictor of survival, patients with N0 disease obtaining a 5-year disease-free survival of 54\% [42].

The EORTC initiated a phase II trial to evaluate the feasibility of trimodal therapy in a multicentre international setting (EORTC 08031) with clearly defined timelines [43]. Patients with pathologically proven mesothelioma of stage cT3N1M0 or less underwent induction chemotherapy consisting of three cycles of cisplatin and pemetrexed followed by EPP within 21-56 days after the last dose of chemotherapy if there was no progressive disease and no unacceptable toxicity. If a patient recovered from surgery and still had a good performance status and was without progressive disease, post-operative radiotherapy was administered, starting 30 days after surgery but within 84 days. In total, 54 Gy were scheduled in 30 daily fractions. The primary end-point of this study was "success of treatment", defined as a patient who had the full trimodal protocol and was alive after 90 days without progressive disease and without grade 3 or 4 toxicity. 57 patients were included and eligible; 42 had EPP (73.7\%) after induction therapy. 90-day mortality was $6.5 \%$. Overall median survival time was 18.4 months and progression-free median survival time 13.9 months (figs 2 and 3). As only 24 (42.1\%) patients met the definition of success, the primary endpoint was not met, demonstrating that this trimodal therapy was feasible but not within the strict time limits defined in this study. A similar phase II tria,l but without predefined time limits, ran at approximately the same time in the USA, including 77 patients from nine institutions. Operative mortality was $7 \%$ and median overall survival time 16.8 months [44]. A comparison between the Swiss, US and EORTC trials is given in table 1.

Although trimodal therapy seemed feasible in selected patients with promising results, the initial outcome of the Mesothelioma and Radical Surgery 1 (MARS 1) trial were less encouraging. As there were concerns over the role of extensive surgery for mesothelioma, MARS 1 was designed as a randomised trial between EPP and no EPP after induction chemotherapy $[45,46]$. To determine whether it was feasible to randomise between EPP or not, a pilot feasibility study was initially performed. During a period of 3 years, 112 patients were evaluated in 11 centres but only 50 (45\%) could be randomised after induction therapy. Randomisation was proven to be feasible in a multicentre setting and the primary end-point was met [47]. However, there were three case fatalities among the 16 patients who underwent EPP, giving a mortality rate of $18.8 \%$ [48]. The median overall survival time for patients undergoing EPP was 14 months, compared with 19 months for those not having EPP, with an adjusted hazard ratio of 2.75 for EPP $(p=0.016)$. The authors concluded that "These data, although limited, suggest that radical surgery in the form of EPP within trimodal therapy offers no benefit and possibly harms patients". So, the proposed surgical procedure in the subsequent MARS 2 trial will be to assess the feasibility of randomisation into P/D and not EPP [49].

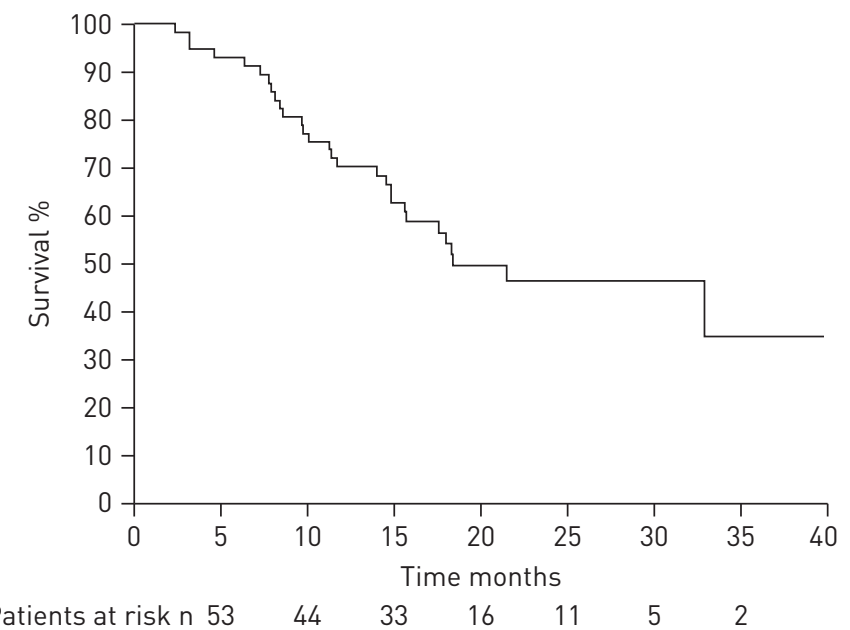

FIGURE 2 Overall survival for all 57 patients who were eligible and started treatment in European Organisation for Research and Treatment of Cancer (EORTC) 0831 phase II trial. Reproduced from [43]. 
FIGURE 3 Progression-free survival for all 57 patients who were eligible and started treatment in European Organisation for Research and Treatment of Cancer (EORTC) 0831 phase II trial. Reproduced from [43].

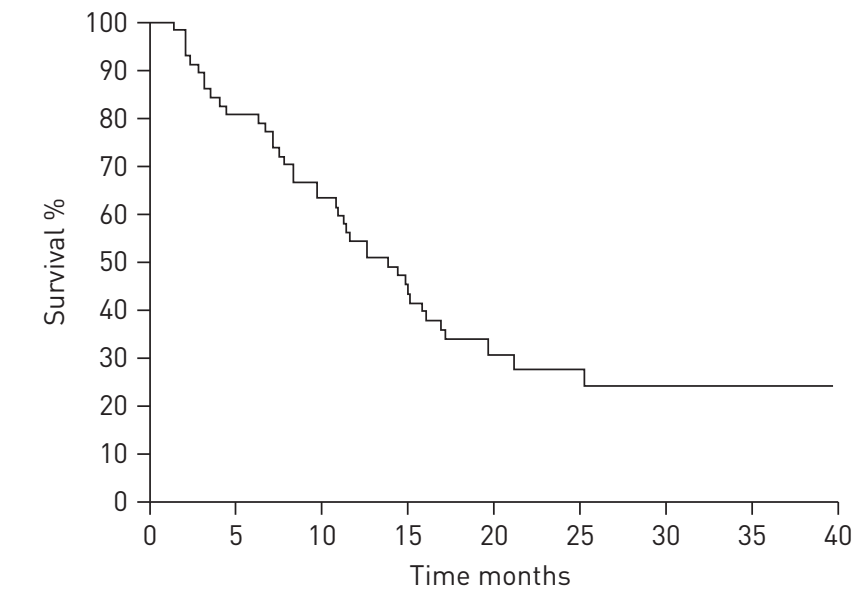

Patients at risk n $\begin{array}{lllllll}46 & 36 & 24 & 10 & 8 & 2 & 2\end{array}$

The results of the pilot MARS trial gave rise to an intense debate in the literature as the mortality of EPP in the MARS was considerably higher than in other centres. Furthermore, the study was not designed, in terms of the sample size, to test the possible benefit of EPP on patient outcome [49].

In a systematic review of EPP for mesothelioma performed in 2010 before the results of the pilot MARS 1 study were published, 34 studies from 26 institutions were evaluated [51]. Median overall survival time after EPP varied from 9.4 to 27.5 months with a 5 -year survival rate from $0 \%$ to $24 \%$. Overall mortality ranged from $0 \%$ to $11.8 \%$ and morbidity from $22 \%$ to $82 \%$. The conclusion of this systematic review was that selected patients might benefit from EPP, especially when combined with induction or adjuvant therapy. Moreover, in a systematic review assessing the safety and efficacy of trimodal treatment, 16 studies were included, one being a randomised controlled trial and five prospective series. The median overall survival time ranged from $12.8-46.9$ months with a perioperative mortality from $0 \%$ to $12.5 \%$ [52]. The authors concluded that trimodal therapy may offer acceptable perioperative outcomes and long-term survival in selected patients treated in specialised centres.

So, where do we go after MARS and what about alternative surgical procedures [53]? To add further confusion to the controversy, $\mathrm{P} / \mathrm{D}$ has emerged as a potentially valid debulking procedure and, in selected cases, as alternative to EPP. Rather surprisingly, in the retrospective study of 663 patients that was

TABLE 1 Comparison of major prospective multicentre phase II trials of radical multimodality treatment in early-stage malignant pleural mesothelioma

SAKK trial [41]

$61 / 6$
Cis-gem $\times 3$
95
$45(74)$
2.2
2.2
$36(59)$
$19.8(14.6-24.5)$
$23.0(16.6-32.9)$
NS
$13.5(10.2-18.8)$
NS

US phase II trial [44]

EORTC $08031[43]$

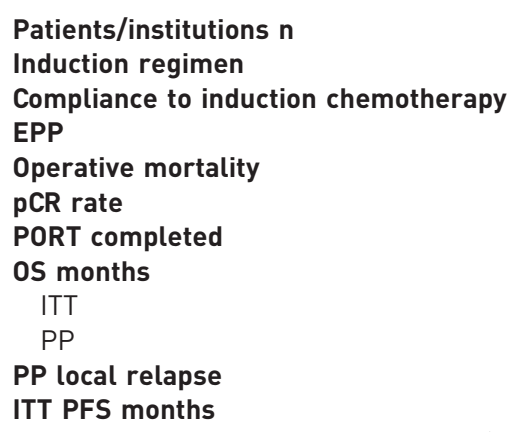

$77 / 9$
Cis-pem $\times 4$
83
$54(70)$
7
5
$40(52)$
$16.8(13.6-23.2)$
$21.9(16.8-29.1)$
$11(28)$
$10.1(8.6-15.0)$
NS

$59 / 11$
Cis-pem $\times 3$
93
$42(74)$
6.5
4.8
$37(65)$
$18.4(15.6-32.9)$
$21.5(17.6-\mathrm{NR})$
$6(16)$
$13.9(10.9-17.2)$
$193(162-220)$

Data are presented as \%, n (\%) or median (95\% CI), unless otherwise stated. SAKK: Swiss Group for Clinical Cancer Research; EORTC: European Organisation for Research and Treatment of Cancer; EPP: extrapleural pneumonectomy; pCR: pathologically complete response; PORT: postoperative radiotherapy; OS: overall survival; ITT: intention to treat; PP: per protocol; PFS: progression-free survival; Cis-gem: cisplatingemcitabine; Cis-pem: cisplatin-pemetrexed; NR: not reached; NS: not stated. 
mentioned previously, multivariate analysis demonstrated a hazard rate of 1.4 for EPP $(\mathrm{p}<0.001)$ compared with $\mathrm{P} / \mathrm{D}$ when controlling for stage, histology, sex and multimodality therapy [33]. Following this finding, more data emerged on the outcome of patients who underwent $\mathrm{P} / \mathrm{D}$, although no controlled studies are currently available comparing both techniques.

Another pivotal, prospective but nonrandomised study compared EPP to P/D performed during a 7-year period [54]. In the EPP group, trimodal therapy was given; 25 patients underwent induction chemotherapy, 22 had EPP and in 17 patients, 54 Gy post-operative radiotherapy was administered. So, the full protocol was successfully accomplished in $68 \%$ of patients. There were 61 patients undergoing P/D with hyperthermic pleural lavage with povidone-iodine as additional intraoperative therapy. Macroscopic complete resection was obtained in $57 \%$ of patients. In 54 patients in this group, prophylactic radiotherapy $(21 \mathrm{~Gy})$ and four to six cycles of adjuvant chemotherapy were administered. 30-day mortality and morbidity for EPP were $4.5 \%$ and $68 \%$, and for P/D $0 \%$ and 28\%, respectively. Median survival time and 5-year survival for EPP were 12.8 months and 9\%, and for P/D 23 months and 30.1\% ( $\mathrm{p}=0.004)$. In multivariable analysis, $\mathrm{P} / \mathrm{D}$, epithelial histology and completeness of resection were significant positive factors related to survival. Therefore, $\mathrm{P} / \mathrm{D}$ not only seems to provide good palliation but also an acceptable local control rate and, possibly, even a better survival. Although this was not a randomised study, the authors concluded that $\mathrm{P} / \mathrm{D}$ is superior to EPP in multimodality management of patients with mesothelioma. However, in an accompanying editorial, WEYANT [55] concluded that P/D cannot yet be considered standard therapy and that "it is too early based on this data to completely abandon EPP altogether".

A recent systematic review on $\mathrm{P} / \mathrm{D}$ concluded that perioperative mortality outcomes were similar between different $\mathrm{P} / \mathrm{D}$ techniques but there was a trend towards higher morbidity and length of hospitalisation for patients undergoing extended P/D [56]. However, overall and disease-free survival appeared to favour extended $\mathrm{P} / \mathrm{D}$ over less aggressive techniques.

Comparison of P/D to EPP remains challenging due to the absence of randomised trials and should be restricted to extended $\mathrm{P} / \mathrm{D}$ procedures only, as both procedures provide macroscopic complete resection.

\section{Summary of current guidelines}

When discussing surgical treatment of mesothelioma with the aim of obtaining a macroscopic complete resection, uniform definitions should be used not only to allow comparison between specific procedures and centres, but also to group data for further analysis [26]. Furthermore a clinical staging system has to be developed, which is reproducible in each centre and matches prognostic features from pathological staging.

In the guidelines of the European Respiratory Society and the European Society of Thoracic Surgeons published in 2010 before the final results of the MARS trial were known, the Task Force concluded that there is little evidence for the efficacy of radical surgery for mesothelioma and it is recommended that radical surgery should only be performed in clinical trials as part of multimodal treatment and restricted to specialised centres [57].

Following a long discussion in multiple forums at the IMIG meeting in 2012, with the main emphasis on surgical input, an agreement was obtained between the attendees on several key issues concerning the treatment of MPM and these are summarised in table 2 [12].

In the Australian guidelines published in July 2013, recommendations 31-33 state that only patients with favourable prognostic features, histology and staging should be referred for consideration of radical treatment involving extensive cytoreductive surgery. The latter should only be used as part of multimodal treatment and restricted to institutions with significant surgical experience and a high volume of cases [58]. Clinical practice point $\mathrm{f}$ indicates that a multidisciplinary team with sufficient experience should provide advice on the suitability of patients for trimodal therapy and the ongoing treatment strategy adopted.

TABLE 2 Summary of key points obtained by consensus at the meeting of International Mesothelioma Interest Group in 2012 [12]

Every patient with presumed mesothelioma should be discussed by a multidisciplinary tumour board

Histology and precise clinical staging should be obtained before initiating therapy

Macroscopic complete resection and control of micrometastatic disease are the main goals of extended surgical treatment within the setting of combined-modality therapy

For operable patients, the choice of surgical procedure (EPP or P/D) largely depends on clinical features, individual surgical evaluation, and expertise of the surgeon and centre where the patient is treated

EPP: extrapleural pneumonectomy; P/D: pleurectomy/decortication. 
Clinical practice point $\mathrm{g}$ mentions that patients whose mesothelioma progresses despite induction (neoadjuvant) chemotherapy should not be offered cytoreductive surgery followed by hemithoracic radiotherapy [58]. These recommendations and clinical practice points represent a nice, up-to-date summary of the current evidence for multimodal therapy for mesothelioma. Thus, the current literature does not allow a definite conclusion on which procedure (EPP or P/D) should be recommended in which stage of the disease and in which combination therapy [53]. The final decision should also be based on the patient's preference after complete information has been provided on the proposed surgical procedure and its possible alternatives.

\section{Further developments}

Due to the overall poor prognosis, several newer therapeutic options are being explored in mesothelioma patients, often in combination with established therapy. As no published, randomised evidence is currently available, no definite recommendations can be made and these newer strategies are still to be further evaluated in prospective trials.

Although the introduction of anti-folate drugs was a major step forward, current results are unlikely to improve further without the addition of targeted or biological agents that interact more specifically with causal pathways in the cellular behaviour of mesothelioma. However, no such agent is currently available for association with induction chemotherapy.

As in non-small cell lung cancer, the role of post-operative radiotherapy in MPM is controversial and mainly based on retrospective series [34]. This additional value of post-operative radiotherapy is being addressed in a Swiss study, in which eligible patients are randomised after EPP to receive either observation or hemithoracic radiotherapy [59]. Inclusion in this study has recently been completed but definite results are awaited.

Preliminary results of intensity-modulated radiotherapy (IMRT) in the adjuvant setting after EPP are particularly promising as good local control was obtained and organs at risk were well protected [60]. However, severe pulmonary toxicity has been reported in recent studies so it should not be recommended outside clinical trials. The role of IMRT with chemotherapy and intact lung is presently being investigated [61]. IMRT may be applied after P/D. The group from the Memorial Sloan-Kettering Cancer Center in New York, NY, USA, described their first experience in 36 patients with a promising 26 months median overall survival time but a substantial toxicity rate including $20 \%$ grade $\geqslant 3$ pneumonitis with one death [62].

A recently described original approach is the application of IMRT before EPP with the so-called SMART (Surgery for Mesothelioma After Radiation Therapy) protocol with promising survival data and without toxicity, especially in the epithelial subgroup, but long-term data are not yet available [63].

As mentioned previously, the addition of hyperthermic pleural lavage with povidone-iodine is feasible but its effect on the rate and time to local recurrence remains unproven [54, 64].

Hyperthermic intraoperative pleural chemotherapy may also be added to cytoreductive surgery to improve locoregional control. In a recent retrospective study, hyperthermic intraoperative chemotherapy with cisplatin was found to yield a shorter time interval to recurrence and increased overall survival [65].

In a recent review, the role of intracavitary therapies for pleural malignancies was evaluated [66]. Intrapleural chemotherapy has only a marginal benefit in pleural malignancies but hyperthermic chemotherapy may prove to be more efficient in combination with maximal tumour debulking. Further pharmacokinetic improvement of intracavitary chemotherapy by binding cisplatin to a fibrin carrier is currently under investigation in a phase I trial [67].

Intrapleural immunotherapies may be most effective in patients with early-stage disease but may prove superior to standard pleurodesis methods to control a recurrent pleural effusion and prolong survival [66]. Intrapleural gene therapy is another promising treatment modality for pleural malignancies but further controlled trials are necessary before it can be applied in routine clinical settings [68].

Recently, intrapleural photodynamic therapy with new photosensitisers has been reconsidered in combination with extended P/D. Results of a pilot trial including 38 patients showed promising results with on overall survival time of 42 months for epithelial histology [69]. However, further confirmation and evaluation of toxicity are necessary before this technique can be applied more widely.

As mentioned before, in view of the results of MARS 1, extended P/D will be the surgical procedure performed in MARS 2 [49]. In this randomised trial, induction chemotherapy followed by extended P/D will be compared to chemotherapy alone. 
In contrast to other tumour types and non-small cell lung cancer, the current induction chemotherapy regimens induce little necrosis and pathologically complete responses, questioning their presumed role in facilitating resection and reducing their efficacy in clearing micrometastatic disease. In the EORTC 1205 randomised phase II trial patients with early-stage mesothelioma will be randomised between induction chemotherapy followed by extended P/D and upfront extended P/D followed by adjuvant chemotherapy [70]. The primary end-point is "success of treatment", defined as a patient who received the full protocol treatment who is still alive after 20 weeks without evidence of progression, relapse or grade $\geqslant 3$ toxicity.

\section{Conclusion}

The role of extensive surgical treatment is currently redefined in mesothelioma patients and no definite guidelines can yet be provided. Macroscopic complete resection should be the aim, obtained either by EPP or extended $\mathrm{P} / \mathrm{D}$, within a multimodal treatment concept. Concerns have arisen over the relatively high mortality and morbidity of EPP compared with extended P/D. Due to the rarity of this tumour, these procedures should only be performed in high-volume thoracic-surgical centres to optimise results. Whether $\mathrm{P} / \mathrm{D}$ yields similar or even better results than EPP remains to be proven by a randomised controlled clinical trial. Moreover, every patient with suspicion of mesothelioma should be discussed by a multidisciplinary tumour board to provide a tailored diagnostic and therapeutic plan. More attention should be paid to quality control and selection criteria of multimodality treatment comprising extensive surgical procedures.

Further prospective trials are required to determine optimal combined modality therapy in mesothelioma. Hopefully, the prospective IASLC database will shed new lights on the predictive and prognostic factors to better determine prognosis and individualise therapy for this dreadful disease.

\section{References}

1 IARC Working Group on the Evaluation of Carcinogenic Risks to Humans. Arsenic, metals, fibres, and dusts. IARC Monogr Eval Carcinog Risks Hum 2012; 100: 11-465.

2 Ambrogi VMT. Clinical and biologic prognostic factors in malignant pleural mesothelioma. Thorac Cancer 2012; 3 : 289-302.

3 Riaz SP, Coupland VH, Luchtenborg M, et al. Mesothelioma incidence projections in South East England. Eur Respir J 2012; 40: 965-968.

4 Rusch VW. A proposed new international TNM staging system for malignant pleural mesothelioma. From the International Mesothelioma Interest Group. Chest 1995; 108: 1122-1128.

Van Schil P. Malignant pleural mesothelioma: staging systems. Lung Cancer 2005; 49: Suppl. 1, S45-S48.

Byrne MJ, Nowak AK. Modified RECIST criteria for assessment of response in malignant pleural mesothelioma. Ann Oncol 2004; 15: 257-260.

7 Labby ZE, Armato SG 3rd, Kindler HL, et al. Optimization of response classification criteria for patients with malignant pleural mesothelioma. J Thorac Oncol 2012; 7: 1728-1734.

8 Armato SG 3rd, Labby ZE, Coolen J, et al. Imaging in pleural mesothelioma: a review of the 11th International Conference of the International Mesothelioma Interest Group. Lung Cancer 2013; 82: 190-196.

9 Frauenfelder T, Tutic M, Weder W, et al. Volumetry: an alternative to assess therapy response for malignant pleural mesothelioma? Eur Respir J 2011; 38: 162-168.

10 Labby ZE, Nowak AK, Dignam JJ, et al. Disease volumes as a marker for patient response in malignant pleural mesothelioma. Ann Oncol 2013; 24: 999-1005.

11 Shersher DD, Liptay MJ. Multimodality treatment of pleural mesothelioma. Surg Oncol Clin N Am 2013; 22: 345-355.

12 Rusch V, Baldini EH, Bueno R, et al. The role of surgical cytoreduction in the treatment of malignant pleural mesothelioma: meeting summary of the International Mesothelioma Interest Group Congress, September 11-14, 2012, Boston, Mass. J Thorac Cardiovasc Surg 2013; 145: 909-910.

13 Rusch VW, Giroux D, Kennedy C, et al. Initial analysis of the international association for the study of lung cancer mesothelioma database. J Thorac Oncol 2012; 7: 1631-1639.

14 Van Schil PE. Treatment of mesothelioma: still a long way to go! Chin J Cancer Res 2013; 25: 132-133.

15 Curran D, Sahmoud T, Therasse P, et al. Prognostic factors in patients with pleural mesothelioma: the European Organization for Research and Treatment of Cancer experience. J Clin Oncol 1998; 16: 145-152.

16 Herndon JE, Green MR, Chahinian AP, et al. Factors predictive of survival among 337 patients with mesothelioma treated between 1984 and 1994 by the Cancer and Leukemia Group B. Chest 1998; 113: 723-731.

17 Francart J, Legrand C, Sylvester R, et al. Progression-free survival rate as primary end point for phase II cancer clinical trials: application to mesothelioma - the EORTC Lung Cancer Group. J Clin Oncol 2006; 24: 3007-3012.

18 Gill RR, Richards WG, Yeap BY, et al. Epithelial malignant pleural mesothelioma after extrapleural pneumonectomy: stratification of survival with CT-derived tumor volume. AJR Am J Roentgenol 2012; 198: 359-363.

19 Opitz IFM, Stahel RA, Weder W. A clinical-based risk score for decision making for surgery after induction chemotherapy in maignant pleural mesothelioma patients. J Clin Oncol 2013; 31: Suppl., 7587.

20 Rusch VW, Giroux D. Do we need a revised staging system for malignant pleural mesothelioma? Analysis of the IASLC database. Ann Cardiothorac Surg 2012; 1: 438-448.

21 Kindler HL. Robust data: the essential foundation of a revised staging system for pleural mesothelioma. $J$ Thorac Oncol 2012; 7: 1623-1624.

22 Meuris K, Hertoghs M, Lauwers P, et al. Subtotal pleurectomy by video-assisted thoracic surgery for metastatic pleuritis. MMCTS 2012; mms008: 10.1093/mmcts/mms008.

23 Halstead JC, Lim E, Venkateswaran RM, et al. Improved survival with VATS pleurectomy-decortication in advanced malignant mesothelioma. Eur J Surg Oncol 2005; 31: 314-320. 
24 Rintoul RCRA, Edwards J, Waller DA, et al. MesoVATS: a multi-centre randomised controlled trial of video-assited thoracoscopic pleurectomy versus talc pleurodesis in malignant pleural mesothelioma. J Thorac Oncol 2013; 8: Suppl. 2, S2-S3.

25 Genc O, Petrou M, Ladas G, et al. The long-term morbidity of pleuroperitoneal shunts in the management of recurrent malignant effusions. Eur J Cardiothorac Surg 2000; 18: 143-146.

26 Sioris T, Sihvo E, Salo J, et al. Long-term indwelling pleural catheter (PleurX) for malignant pleural effusion unsuitable for talc pleurodesis. Eur J Surg Oncol 2009; 35: 546-551.

27 Rice D, Rusch V, Pass $\mathrm{H}$, et al. Recommendations for uniform definitions of surgical techniques for malignant pleural mesothelioma: a consensus report of the international association for the study of lung cancer international staging committee and the international mesothelioma interest group. J Thorac Oncol 2011; 6: 1304-1312.

28 Singhal S, Kaiser LR. Malignant mesothelioma: options for management. Surg Clin N Am 2002; 82: 797-831.

29 Vogelzang NJ, Rusthoven JJ, Symanowski J, et al. Phase III study of pemetrexed in combination with cisplatin versus cisplatin alone in patients with malignant pleural mesothelioma. J Clin Oncol 2003; 21: 2636-244.

30 van Meerbeeck JP, Gaafar R, Manegold C, et al. Randomized phase III study of cisplatin with or without raltitrexed in patients with malignant pleural mesothelioma: an intergroup study of the European Organisation for Research and Treatment of Cancer Lung Cancer Group and the National Cancer Institute of Canada. J Clin Oncol 2005; 23: 6881-6889.

31 Green J, Dundar Y, Dodd S, et al. Pemetrexed disodium in combination with cisplatin versus other cytotoxic agents or supportive care for the treatment of malignant pleural mesothelioma. Cochrane Database Syst Rev 2007; 1: CD005574.

32 Sugarbaker DJ, Flores RM, Jaklitsch MT, et al. Resection margins, extrapleural nodal status, and cell type determine postoperative long-term survival in trimodality therapy of malignant pleural mesothelioma: results in 183 patients. J Thorac Cardiovasc Surg 1999; 117: 54-63.

33 Flores RM, Pass HI, Seshan VE, et al. Extrapleural pneumonectomy versus pleurectomy/decortication in the surgical management of malignant pleural mesothelioma: results in 663 patients. J Thorac Cardiovasc Surg 2008; 135: 620-626.

34 Rusch VW, Rosenzweig K, Venkatraman E, et al. A phase II trial of surgical resection and adjuvant high-dose hemithoracic radiation for malignant pleural mesothelioma. J Thorac Cardiovasc Surg 2001; 122: 788-795.

35 Boutin C, Rey F, Viallat JR. Prevention of malignant seeding after invasive diagnostic procedures in patients with pleural mesothelioma. A randomized trial of local radiotherapy. Chest 1995; 108: 754-758.

36 Bydder S, Phillips M, Joseph DJ, et al. A randomised trial of single-dose radiotherapy to prevent procedure tract metastasis by malignant mesothelioma. Br J Cancer 2004; 91: 9-10.

37 Lee C, Bayman N, Swindell R, et al. Prophylactic radiotherapy to intervention sites in mesothelioma: a systematic review and survey of UK practice. Lung Cancer 2009; 66: 150-156.

38 O'Rourke N, Garcia JC, Paul J, et al. A randomised controlled trial of intervention site radiotherapy in malignant pleural mesothelioma. Radiother Oncol 2007; 84: 18-22.

39 Davies HE, Musk AW, Lee YC. Prophylactic radiotherapy for pleural puncture sites in mesothelioma: the controversy continues. Curr Op Pulm Med 2008; 14: 326-330.

40 Ung YC, Yu E, Falkson C, et al. The role of radiation therapy in malignant pleural mesothelioma: a systematic review. Radiother Oncol 2006; 80: 13-18.

41 Weder W, Stahel RA, Bernhard J, et al. Multicenter trial of neo-adjuvant chemotherapy followed by extrapleural pneumonectomy in malignant pleural mesothelioma. Ann Oncol 2007; 18: 1196-1202.

42 de Perrot M, Feld R, Cho BC, et al. Trimodality therapy with induction chemotherapy followed by extrapleural pneumonectomy and adjuvant high-dose hemithoracic radiation for malignant pleural mesothelioma. J Clin Oncol 2009; 27: 1413-1418.

43 Van Schil PE, Baas P, Gaafar R, et al. Trimodality therapy for malignant pleural mesothelioma: results from an EORTC phase II multicentre trial. Eur Respir J 2010; 36: 1362-1369.

44 Krug LM, Pass HI, Rusch VW, et al. Multicenter phase II trial of neoadjuvant pemetrexed plus cisplatin followed by extrapleural pneumonectomy and radiation for malignant pleural mesothelioma. J Clin Oncol 2009; 27: 3007-3013.

45 Treasure T, Utley M. Mesothelioma: benefit from surgical resection is questionable. J Thorac Oncol 2007; 2: 885-886.

46 Treasure T, Tan C, Lang-Lazdunski L, et al. The MARS trial: mesothelioma and radical surgery. Interac Cardiovas Thoracic Surg 2006; 5: 58-59.

47 Treasure T, Waller D, Tan C, et al. The Mesothelioma and Radical Surgery randomized controlled trial: the MARS feasibility study. J Thorac Oncol 2009; 4: 1254-1258.

48 Treasure T, Lang-Lazdunski L, Waller D, et al. Extra-pleural pneumonectomy versus no extra-pleural pneumonectomy for patients with malignant pleural mesothelioma: clinical outcomes of the Mesothelioma and Radical Surgery (MARS) randomised feasibility study. Lancet Oncol 2011; 12: 763-772.

49 Steele JP. Randomised trial of extended pleurectomy and decortication (EPD) with pemetrexed and platinum chemotherapy versus pemetrexed and platinum alone in patients with malignant pleural mesothelioma ("MARS2"). Proc IMIG 2012; 2012: P3.22.

50 Weder W, Stahel RA, Baas P, et al. The MARS feasibility trial: conclusions not supported by data. Lancet Oncol 2011; 12: 1093-1094.

51 Cao CQ, Yan TD, Bannon PG, et al. A systematic review of extrapleural pneumonectomy for malignant pleural mesothelioma. J Thorac Oncol 2010; 5: 1692-1703.

52 Cao C, Tian D, Manganas C, et al. Systematic review of trimodality therapy for patients with malignant pleural mesothelioma. Ann Cardiothorac Surg 2012; 1: 428-437.

53 Hiddinga BI, van Meerbeeck JP. Surgery in mesothelioma-where do we go after MARS? J Thorac Oncol 2013; 8: 525-529.

54 Lang-Lazdunski L, Bille A, Lal R, et al. Pleurectomy/decortication is superior to extrapleural pneumonectomy in the multimodality management of patients with malignant pleural mesothelioma. J Thorac Oncol 2012; 7: 737-743.

55 Weyant MJ. Is it time to consider pleurectomy and decortication as the only surgical treatment for malignant pleural mesothelioma? J Thorac Oncol 2012; 7: 629-630. 
Cao C, Tian DH, Pataky KA, et al. Systematic review of pleurectomy in the treatment of malignant pleural mesothelioma. Lung Cancer 2013; 81: 319-327.

57 Scherpereel A, Astoul P, Baas P, et al. Guidelines of the European Respiratory Society and the European Society of Thoracic Surgeons for the management of malignant pleural mesothelioma. Eur Respir J 2010; 35: 479-495.

58 Asbestos Diseases Research Institute. Guidelines for the diagnosis and treatment of malignant pleural mesothelioma. www.adri.org.au/documents/Guidelines_for_the_diagnosis_and_treatment_of_malignant_pleural_mesothelioma.pdf Date last accessed: 2013. Date last updated: July 2013.

59 Swiss Group for Clinical Cancer Research. Project Group Lung Cancer. http://sakk.ch/en/about-sakk/organization/ research-groups/project-group-lung-cancer/ Date last accessed: January 25, 2014. Date last updated: 2014.

60 Rice DC, Stevens CW, Correa AM, et al. Outcomes after extrapleural pneumonectomy and intensity-modulated radiation therapy for malignant pleural mesothelioma. Ann Thorac Surg 2007; 84: 1685-1692.

61 Memorial Sloan-Kettering Cancer Center. Study Using Chemotherapy +/- Pleurectomy/Decortication Followed By Intensity Modulated Radiation Therapy. www.clinicaltrials.gov/ct2/show/NCT00715611 Date last accessed: January 25, 2014. Date last updated: January 17, 2014

62 Rosenzweig KE, Zauderer MG, Laser B, et al. Pleural intensity-modulated radiotherapy for malignant pleural mesothelioma. Int J Radiat Oncol Biol Phys 2012; 83: 1278-1283.

63 De Perrot MMO, Feld R, Leighl N, et al. Five-year experience with accelerated induction hypofractionated hemithoracic intensity modulated radiation therapy (IMRT) followed by extrapleural pneumonectomy (EPP) for malignant pleural mesothelioma (MPM). J Thorac Oncol 2013; 8: Suppl. 2, S345-S346.

64 Lang-Lazdunski L, Bille A, Belcher E, et al. Pleurectomy/decortication, hyperthermic pleural lavage with povidoneiodine followed by adjuvant chemotherapy in patients with malignant pleural mesothelioma. J Thorac Oncol 2011; 6: $1746-1752$

65 Sugarbaker DJ, Gill RR, Yeap BY, et al. Hyperthermic intraoperative pleural cisplatin chemotherapy extends interval to recurrence and survival among low-risk patients with malignant pleural mesothelioma undergoing surgical macroscopic complete resection. J Thorac Cardiovasc Surg 2013; 145: 955-963.

66 Haas AR, Sterman DH. Intracavitary therapeutics for pleural malignancies. Clin Chest Med 2013; 34: 501-513

67 Opitz ILO, Meerang M, Friess M, et al. Intracavitary cisplatin-fibrin chemotherapy after resection for malignant pleural mesothelioma patients (Influence-meso) - preliminary results. J Thorac Oncol 2013; 8: Suppl. 2 , S343.

68 Sterman DH, Albelda SM. Advances in the diagnosis, evaluation, and management of malignant pleural mesothelioma. Respirology 2005; 10: 266-283.

69 Friedberg JS. Radical pleurectomy and photodynamic therapy for malignant pleural mesothelioma. Ann Cardiothorac Surg 2012; 1: 472-480.

70 Lang-Lazdunski L, Van Meerbeeck JP, Vermassen F. EORTC randomised phase II study of extended pleurectomy/ decortication (e-P/D) preceded or followed by (neo-)adjuvant chemotherapy in patients with early stage malignant pleural mesothelioma (MPM). Proc IMIG 2012; 2012: P4.14. 